\title{
DECOMPOSITION SOLUTION FOR DUFFING AND \\ VAN DER POL OSCILLATORS
}

\author{
G. ADOMIAN \\ Center for Applied Mathematics \\ University of Georgia \\ Athens, Georgia 30602 U.S.A. \\ (Received February 13, 1986)
}

ABSTRACT. The decomposition method is applied to solve the Duffing and Van der Pol oscillators without customary restrictive assumptions [1-4] and without resort to perturbation methods.

1980 AMS MATHEMATICS SUBJECT CLASSIFICATION CODES. 36620

KEY WORDS AND PHRASES. Duffing oscillator, Van der Pol oscillator, nonlinear, stochastic, decomposition.

1. INTRODUCTION

The Duffing equation is written

$$
\ddot{y}+\alpha \dot{y}+\beta y+\gamma y^{3}=x(t)
$$

The Van der Pol equation can be written

$$
\ddot{y}+\alpha \dot{y}+\beta y+\gamma(d / d t) y^{3}=x(t)
$$

(If $\alpha=-\xi, \beta=1, \gamma=\xi / 3$, we have the form usually given.) Write $L=d^{2} / d t^{2}, \quad R=\alpha(d / d t)+B, \quad N y=\gamma y^{3}$ in (1.1) and $\gamma(d / d t) y^{3}$ in (1.2) Thus both are written

$$
L y+R y+N y=x(t)
$$

in the standard form for the decomposition method [1-3] where $L^{-1}$ is the two-fold definite integral from 0 to $t$. Then,

$$
L y=x(t)-R y-N y \text {. }
$$
Assuming initial conditions $y(0), y^{\prime}(0)$ are specified, let $y=\sum_{n=0}^{\infty} y_{n}$ and
define $y_{0}$ by

$$
y_{0}=y(0)+t y^{\prime}(0)+L^{-1} x(t)
$$

Then

$$
y_{n+1}=-L^{-1} \alpha(d / d t) y_{n}-L^{-1} B y_{n}-L^{-1}[N y]
$$

for $n \geq 0$. 


\section{SOLUTION OF THE PROBLEM}

To get computable solutions, we need only substitute for $N y$ the sum $\gamma \sum_{n=0}^{\infty} A_{n}$ for the Duffing case and $r(d / d t) \sum_{n=0}^{\infty} A_{n}$ for the Van der Pol case where the $A_{n}$ are Adomian's polynomials [1-5] generated for the nonlinear term $y^{3}$ and representing it exactly in a rapidly converging series [1-5].

$$
\begin{aligned}
& A_{0}=y_{0}^{3} \\
& A_{1}=3 y_{0}^{2} y_{1} \\
& A_{2}=3 y_{0}^{2} y_{2}+3 y_{0} y_{1}^{2} \\
& A_{3}=3 y_{0}^{2} y_{3}+6 y_{0} y_{1} y_{2}+y_{1}^{3} \\
& A_{4}=3 y_{0}^{2} y_{4}+3 y_{0} y_{2}^{2}+6 y_{0} y_{1} y_{3}+3 y_{1}^{2} y_{2} \\
& A_{5}=3 y_{0}^{2} y_{5}+6 y_{0} y_{1} y_{4}+6 y_{0} y_{2} y_{3}+3 y_{1}^{2} y_{3}+3 y_{1} y_{2}^{2}
\end{aligned}
$$

The deterministic problem is now solved since all components of $y$ are determined. We use an n-term approximation $\phi_{n}=\sum_{i=0}^{n} y_{i}$ which, because of the rapid convergence, is generally sufficient with a very smail $n$ (say half a dozen or so terms) but easily carried as far as necessary since the integrals do not involve difficult Green's functions. Convergence has been previously established by Adomian $[2,5]$ and has been shown [2] to be quite rapid.

For the stochastic case [2], none of the usual approximations of statistical linearization are necessary. The $x(t)$ need not be stationary nor Gaussian nor delta-correlated. Further $\alpha, \beta, \gamma$ and the initial conditions can be stochastic. No "smallness" assumptions are necessary for the stochastic processes and the nonlinearities. No linearization is used. We can allow $\alpha_{\infty}=\langle\alpha\rangle+\xi, \beta=\langle\beta\rangle+\eta$, $\gamma=\langle\gamma\rangle+\sigma$ and write $L y=x-\langle\alpha\rangle(d / d t) y-\langle\beta\rangle y-\langle\gamma\rangle \sum_{n=0}^{\infty} A_{n}-\xi(d / d t) y-n y-$ $\sigma \sum_{n=0}^{\infty} A_{n}$ and proceed as before with $y=\sum_{n=0}^{\infty} y_{n}$.

The result is a stochastic series from which statistics are obtained without the problems of statistical separability of quantities such as $\langle R y\rangle$ where $R=\xi \mathrm{d} / \mathrm{dt}-n$ which normally require closure approximations.

\section{REFERENCES}

1. ADOMIAN, G. Stochastic Systems, Academic Press, 1983.

2. ADOMIAN, G. Nonlinear Stochastic Operator Equations, Academic Press, 1986.

3. BELLMAN, R.E., ADOMIAN, G. Nonlinear Partial Differential Equations - New Methods for Their Treatment and Applications, Reidel, 1985.

4. ADOMIAN, G., RACH, R. Anharmonic Oscillator Systems, J. Math. Anal. and Applic. 91, no. 1 (January 1983), 229-236.

5. ADOMIAN, G. Convergent Series Solution of Nonlinear Equations, J. Comput. and App. Math. 11, no. 2 (October 1984), 225-230.

6. BONZANI, I. On a Class of Nonlinear Stochastic Dynamical Systems: Analysis of the Transient Behavior, J. Math. Anal. and Applic., to appear. 


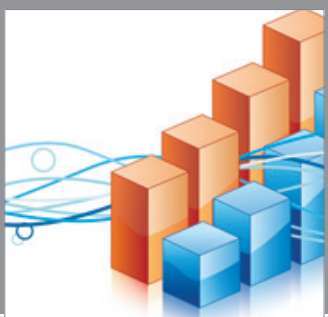

Advances in

Operations Research

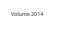

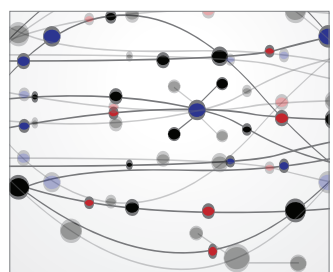

\section{The Scientific} World Journal
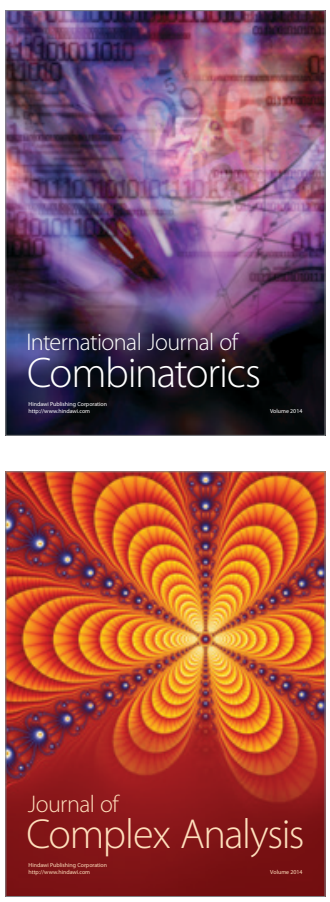

International Journal of

Mathematics and

Mathematical

Sciences
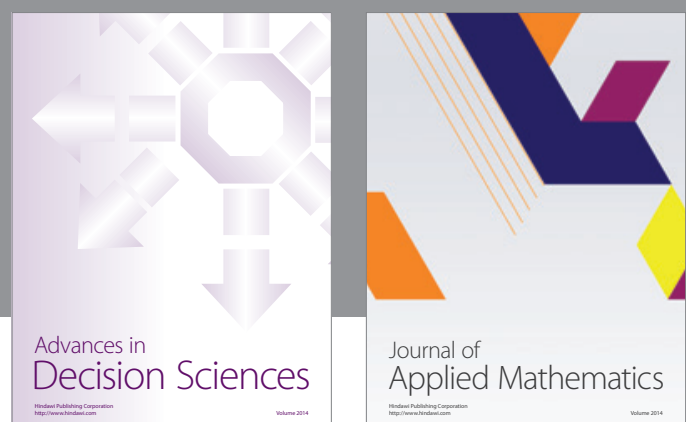

Journal of

Applied Mathematics
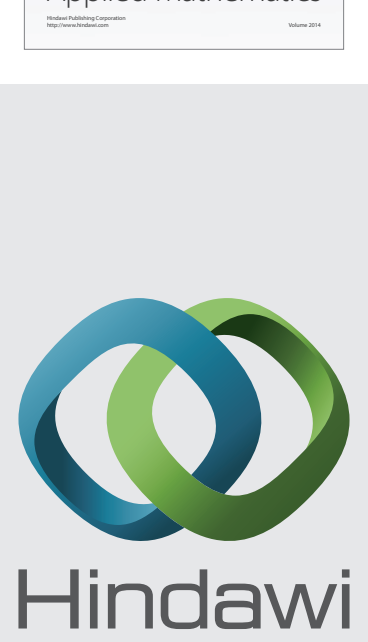

Submit your manuscripts at http://www.hindawi.com
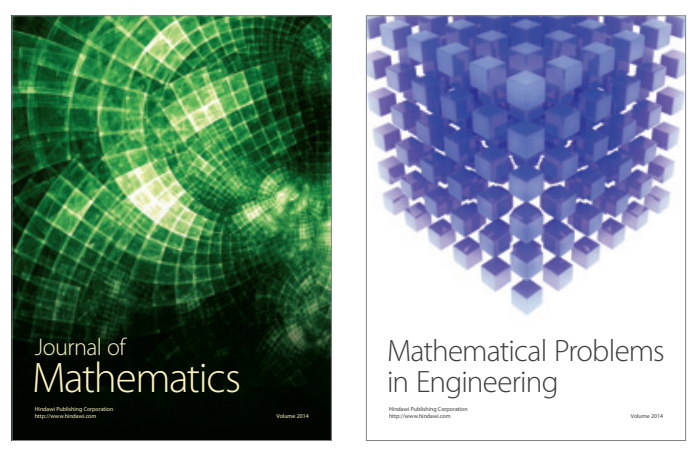

Mathematical Problems in Engineering
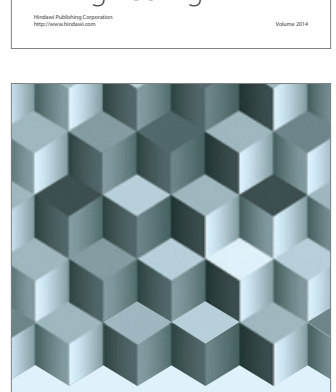

Journal of

Function Spaces
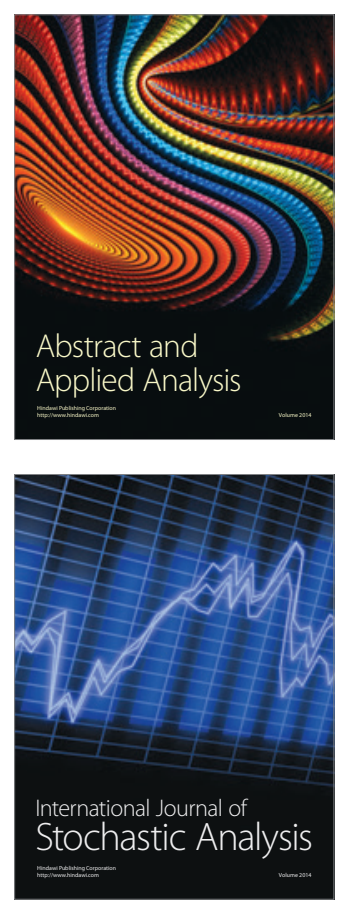

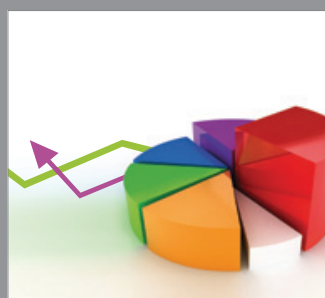

ournal of

Probability and Statistics

Promensencen
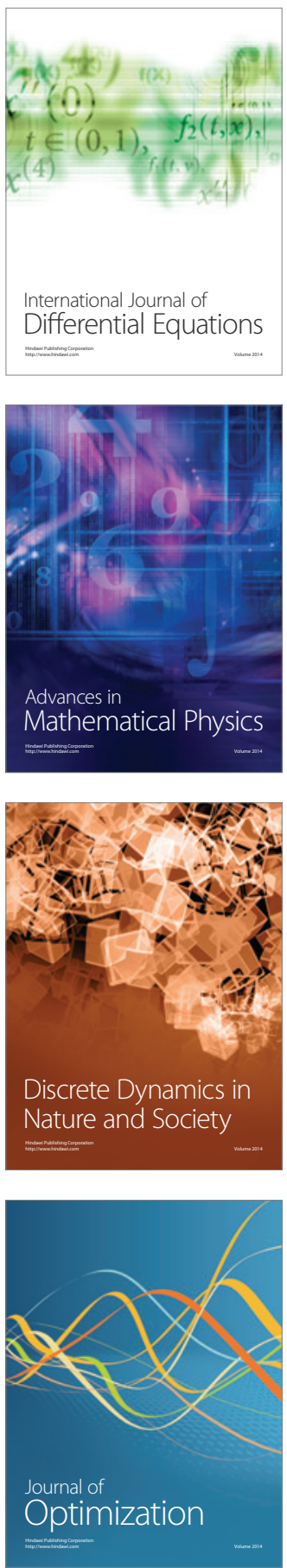